\title{
A Randomized Algorithm for Gossiping in Radio Networks
}

\author{
Marek Chrobak \\ Department of Computer Science, University of California, Riverside, California 92521
}

Leszek Gạsieniec

Department of Computer Science, University of Liverpool, Liverpool L69 7ZF United Kingdom

\author{
Wojciech Rytter \\ Instytut Informatyki, Uniwersytet Warszawski, Banacha 2, 02-097, Warszawa, Poland; Department of Computer \\ Science, University of Liverpool, Liverpool L69 7ZF, United Kingdom
}

\begin{abstract}
We present an $O\left(n \log ^{4} n\right)$-time randomized algorithm for gossiping in radio networks with unknown topology. This is the first algorithm for gossiping in this model whose running time is only a polylogarithmic factor away from the optimum. The fastest previously known (deterministic) algorithm for this problem works in time $O\left(n^{3 / 2} \log ^{2} n\right)$. ( 2004 Wiley Periodicals, Inc.
\end{abstract}

Keywords: radio networks; gossiping; randomized algorithms

\section{INTRODUCTION}

The two classical problems of disseminating information in computer networks are broadcasting and gossiping. In broadcasting, we want to distribute a given message from a distinguished source node to all other nodes in the network. In gossiping, each node $v$ in the network initially contains a message $m_{v}$, and we wish to distribute each message $m_{v}$ to all nodes in the network. In both problems, we would like to minimize the time needed to complete the task.

In radio networks, a message transmitted by a processor is sent to all processors within its range. The range relation is represented by a graph of nodes with directed edges between them. All processors work synchronously, and if a processor $u$ transmits a message $m$ at time step $t$, the

Received September 2000; accepted September 2003

Correspondence to: M. Chrobak; e-mail: marek@cs.ucr.edu

Contract grant sponsor: EPSRC; contract grant numbers: GR/N09077; GR/R85921

Contract grant sponsor: NSF; contract grant numbers: CCR-9988360; CCR-0208856

(C) 2004 Wiley Periodicals, Inc. message reaches each neighbor $v$ of $u$ at the same time step. Node $v$ will successfully receive $m$ only if $u$ is the only processor, among those whose range contains $v$, that transmits at time $t$. Since the communication links are unidirectional, there is no feedback mechanism in the network (see, e.g., [21]), and, thus, in general, a node does not know for certain whether its transmissions were successful. Further, we assume that collisions cannot be resolved or detected, that is, if messages from two or more processors reach $v$ at time $t, v$ does not receive any message and it does not know that the collision occurred. This is motivated by situations when message collisions are difficult to distinguish from background noise, in which protocols that do not depend on the accuracy of the collision detection mechanism (see [13, 15]) will be more reliable.

We focus on gossiping algorithms that do not use any information about network topology. Such topology-independent algorithms are useful in networks with mobile users or unstable topologies, since, then, one does not need to change or reconfigure the protocol after topology changes. As long as the network is strongly connected and no changes occur during the actual execution of the algorithm, the task of gossiping will complete successfully. The strong connectivity assumption is necessary for gossiping to be meaningful.

Past Work

Most of the previous work on radio networks focused on broadcasting. If the topology of the network is known to all processors, Gaber and Mansour [14] showed that broadcasting can be achieved in time $O\left(D+\log ^{5} n\right)$, where $D$ is the network diameter. Diks et al. [12] gave efficient broadcast- 
ing algorithms for special types of known networks. It is also known that computing an optimal broadcast schedule for a given network is NP-hard, even for points in the plane, where the graph is induced by node ranges, see $[6,8,27]$.

For networks with unknown topology, Bar-Yehuda et al. [3] gave randomized algorithm that achieves broadcast in expected time $O\left(D \log n+\log ^{2} n\right)$. This is very close to the lower bound of $\Omega(D \log (n / D))$, by Kushilevitz and Mansour [19], and it matches this lower bound for a wide range of depth values, for example, when $D=\Theta\left(n^{1-\epsilon}\right)$, for any $\epsilon>0$. Further, if $D$ is a constant, it also matches the lower bound of $\Omega\left(\log ^{2} n\right)$ for constant diameter networks, obtained by Alon et al. [1].

In the deterministic case, Bar-Yehuda et al. [3] gave an $\Omega(n)$ lower bound for constant-diameter networks. For general networks, the best currently known lower bound of $\Omega(n$ $\log n$ ) was obtained by Bruschi and del Pinto [5] and, independently, by Chlebus et al. [7]. In [7], the authors also presented a broadcast algorithm with time complexity $O\left(n^{11 / 6}\right)$ - the first subquadratic upper bound. This upper bound was later improved to $O\left(n^{5 / 3} \log ^{3} n\right)$ by De Marco and Pelc [11]. Chlebus et al. [8] developed several broadcasting algorithms, including one with time complexity $O\left(n^{3 / 2}\right)$. In an independent work, using a probabilistic construction, Peleg [24] gave an $O\left(n^{3 / 2} \sqrt{\log n}\right)$ upper bound. Recently, Chrobak et al. [10] presented a deterministic algorithm for broadcasting with time complexity $O(n$ $\left.\log ^{2} n\right)$, thus nearly matching the lower bound of $\Omega(n \log n)$ from [5, 7]. As in [24], this broadcasting algorithm was constructed using a probabilistic argument.

The problem of gossiping has been intensely studied in various network models (see, e.g., [16]), but relatively little work has been done for radio networks. Ravishankar and Singh [25, 26] studied gossiping algorithms for some restricted topologies, including paths and rings, under probabilistic assumptions on the spatial distribution of nodes. In our previous work, [10], we developed a deterministic algorithm for gossiping with time complexity $O\left(n^{3 / 2} \log ^{2} n\right)$, which, to our knowledge, is the only subquadratic algorithm for gossiping in radio networks with unknown topology.

The case of undirected graphs, that is, networks with bidirectional links, is known to be easier. For this case, Chlebus et al. [7] showed an algorithm that can achieve gossiping in linear time. (They presented it as an algorithm for broadcasting, but it can be easily extended to perform gossiping.) Even if the messages are restricted to have size $O(\log n)$, the work of Bar-Yehuda et al. [4] implies that, using randomization, gossiping in undirected graphs can be achieved in expected time $O\left(n \log ^{2} n\right)$.

The results from [4] underscores the difference between the directed and undirected networks, since, for directed graphs, it is known that if the messages have size $O(\log n)$ then any gossiping algorithm must take time $\Omega\left(n^{2}\right)$, even if the input graph is revealed (see [9]). In this paper (as well as in [10]), we make no assumptions on the message size.

\section{Our Results}

We give a randomized $O\left(n \log ^{4} n\right)$-time algorithm for gossiping in radio networks with unknown topology. Our basic algorithm is Monte Carlo and it has the following performance characteristics: For any $0<\epsilon<1$, in time $O(n$ $\left.\log ^{3} n \log (n / \epsilon)\right)$ it completes gossiping with probability at least $1-\epsilon$. This easily yields a Las Vegas algorithm with expected running time $O\left(n \log ^{4} n\right)$.

\section{PRELIMINARIES}

\section{Radio Networks}

A radio network (see $[3,8]$ ) is defined as an $n$-node directed graph whose nodes are assigned unique identifiers from the set $\{1,2, \ldots, n\}$. Throughout the paper, for gossiping to be meaningful, we assume that the network is strongly connected. If there is an edge from $u$ to $v$, then we say that $v$ is an out-neighbor of $u$ and $u$ is an in-neighbor of $v$.

Initially, each node $v$ contains a message $m_{v}$ and has no other information. The time is divided into discrete time steps. All nodes start simultaneously, have access to a common clock, and work synchronously. (As noted by Peleg [24], the assumption about a common clock is not necessary.) At any time step, a node can be in one of two states: the receiving state or the transmitting state. A gossiping algorithm is a protocol that, for each identifier $i d$ and for each time step $t$, given all past messages received by $i d$, specifies the state of $i d$ at time $t$. If $i d$ transmits at time $t$, the protocol specifies the message. A message $m$ transmitted at time $t$ from a node $u$ is sent instantly to all its out-neighbors. However, an out-neighbor $v$ of $u$ receives $m$ at time step $t$ only if $v$ is in the receiving state and if no collision occurred, that is, if the other in-neighbors of $v$ do not transmit at time $t$ at all. Further, collisions cannot be distinguished from background noise. If $v$ does not receive any message at time $t$, it knows that either none of its in-neighbors transmitted at time $t$ or that at least two did, but it does not know which of these two events occurred.

The running time of a gossiping algorithm is the smallest $t$ such that, for any strongly connected network topology and for any assignment of identifiers to the nodes, each node receives all messages $m_{v}$ no later than at step $t$.

\section{Simplifying Assumptions}

For clarity of presentation, we will present our algorithms as if the nodes knew $n$, the size of the network. This assumption can be eliminated by a standard doubling technique (see $[7,8]$ ) that works as follows: We organize the computation into phases, and we modify a given algorithm so that in phase $i$ only nodes with labels at most $2^{i}$ participate in the algorithm. This does not change the asymptotic running time.

Further, we will also assume throughout the paper that $n$ 
is a power of 2 . For other $n$, the processors can execute the algorithm for the nearest power of 2 larger than $n$, without changing the asymptotic running time.

\section{Notation}

By $V$, we denote the set of nodes, and individual nodes are denoted by letters $u, v, \ldots$ Messages are denoted by the letter $m$, possibly with indices. The message originating from a node $v$ is denoted by $m_{v}$. The whole set of initial messages is $M=\left\{m_{v}: v \in V\right\}$. During the computation, each node $v$ will store a set of messages $M_{v}$ that have been received by $v$ so far. Initially, $M_{v}=\left\{m_{v}\right\}$. Without loss of generality, whenever a node is in the transmit mode, we can assume that it transmits the whole $M_{v}$. This is achieved by a procedure denoted transmit $\left(M_{v}\right)$. Procedure receive( ) puts $v$ in the receive mode and it returns the received message or null if no message has been received.

\section{LIMITED BROADCAST}

One component of our algorithm is a procedure for limited broadcasting. Given an integer $k$ and a node $v$, the goal of limited broadcasting is to send the message $M_{v}$ to at least $k$ nodes in the network. We refer to $v$ as the source node or the node that initiates the broadcast and to $M_{v}$ as the source message.

In [10], the broadcasting algorithm is defined by a sequence $\bar{S}=S_{0} S_{1} \ldots$ of subsets of $\{1,2, \ldots, n\}$. We refer to the sets $S_{t}$ as transmission sets. At time $t$, any node $v$ that has already received the source message checks whether $v$ $\in S_{t}$. If so, $v$ transmits the message; otherwise, $v$ is quiet. We modify the algorithm from [10], so that it performs the broadcasting procedure for only $O\left(k \log ^{2} n\right)$ steps. Below, we appropriately refine the correctness proof, since the proof from [10] is not sufficient for our purpose.

The pseudocode for the algorithm executed by each node is given below. Each node $v$ has a Boolean flag active ${ }_{v}$ that indicates whether $v$ is active, that is, whether $v$ has received a source message. Each iteration of the for-loop lasts one time step. The value of constant $\gamma$ will be determined later.

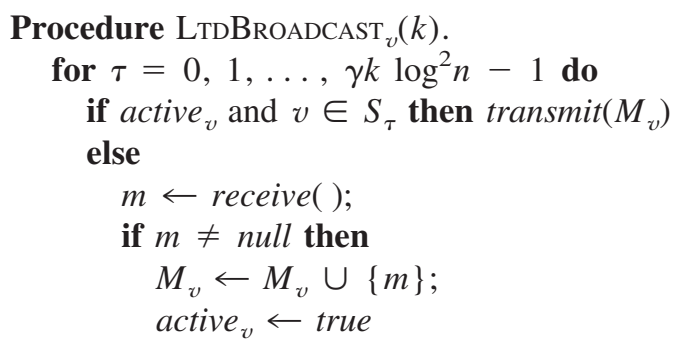

Lemma 1. Assume that, initially, exactly one node $u$ is active and that all nodes $v$ begin executing $\operatorname{LTDBROADCAST}_{y}(k)$ simultaneously. Then, for some constant $\gamma$ independent of $k$, after the computation is complete, at least $k$ nodes will receive the message from $u$.
Proof. Without loss of generality, assume that $n \geq 2$. We first explain the construction of the transmission sets from [10].

It was shown in [10] that there is a constant $\alpha$ such that for each $j=0, \ldots, \log n$ there is a family $\bar{S}_{j}=\left(S_{j, 0}\right.$, $\left.S_{j, 1}, \ldots, S_{j, a_{j}-1}\right)$ of $a_{j}=\alpha 2^{j} \log n$ sets with the following property:

(*) For any two disjoint sets $X, Y$ with $2^{j-1} \leq|X| \leq 2^{j}$ and $|Y| \leq 2^{j}$ there exists a set $S_{j, i}$ in $\bar{S}_{j}$ such that $\left|X \cap S_{j, i}\right|$ $=1$ and $Y \cap S_{j, i}=\varnothing$.

Let $\gamma=12 \alpha+2$. The sequence $\bar{S}$ consists of stages, with each stage, except possibly the last one, having $\log n$ +1 steps. Note that the number of stages is $\left\lceil\gamma k \log ^{2} n /(\log \right.$ $n+1)\rceil \geq 6 \alpha k \log n+1$. The transmission set at the $j$ th step of stage $s$, that is, $S_{\tau}$ for $\tau=s(\log n+1)+j$, is $S_{j, s \bmod a_{j}}$.

Among the active nodes, we distinguish two types of nodes: frontier nodes, which still have inactive out-neighbors, and inner nodes, which do not. If there is a time step $t<\gamma k \log ^{2} n$ when there are $k$ or more frontier nodes, then the lemma holds trivially. So, from now on, we assume that, at each step, the number of frontier nodes is less than $k$.

We define a sequence of stages $s_{0}=0, s_{1}, \ldots, s_{l+1}$ $=6 \alpha k \log n$, where $1 \leq s_{c+1}-s_{c} \leq 2 \alpha k \log n$ for all $c$. Denote by $i_{c}$ and $f_{c}$ the number of inner and frontier nodes, respectively, when stage $s_{c}$ is about to start. We will choose $s_{1}, \ldots, s_{l}$ so that the following invariant holds for each $c \leq l$ :

$$
2 i_{c}+f_{c} \geq \frac{s_{c}}{2 \alpha \log n}
$$

Given (1), we can prove the lemma as follows: The number of nodes that have received the message when stage $s_{l+1}$ ends is at least

$$
i_{l}+f_{l} \geq \frac{1}{2}\left(2 i_{l}+f_{l}\right) \geq \frac{s_{l}}{4 \alpha \log n} \geq \frac{s_{l+1}-2 \alpha k \log n}{4 \alpha \log n}=k .
$$

So, it is sufficient to construct $s_{1}, \ldots, s_{l}$ that satisfy (1). We define these stages inductively. For $c=0$, we have $s_{0}$ $=0, i_{0}=0$, and $f_{0}=1$, so (1) holds. Suppose that we have determined some $s_{c}$. If $s_{c}>4 \alpha k \log n$, set $l=c$ and we are done. Otherwise, we proceed as follows:

Let $F$ be the set of frontier nodes at the beginning of stage $s_{c}$, and let $g$ be such that $2^{g-1} \leq|F|=f_{c}<2^{g}$. For each $j=1, \ldots, g$, let $Y_{j}$ be the set of nodes that received the message in stages $s_{c}, s_{c}+1, \ldots, s_{c}+a_{j}-1$ (but were inactive when stage $s_{c}$ started). We have two subcases:

CAse 1. There is $j$ for which $\left|Y_{j}\right| \geq 2^{j}$. In this case, take $s_{c+1}=s_{c}+a_{j}$. At least $\left|Y_{j}\right|$ new nodes received the message, so 
$2 i_{c+1}+f_{c+1} \geq 2 i_{c}+f_{c}+\left|Y_{j}\right| \geq \frac{s_{c}}{2 \alpha \log n}+2^{j} \geq \frac{s_{c+1}}{2 \alpha \log n}$.

Case 2. For each $j$, we have $\left|Y_{j}\right| \leq 2^{j}$. We show that, in this case, all nodes in $F$ will become inner after $a_{g}$ stages.

Fix any node $v$ that is inactive when stage $s_{c}$ starts and whose set $X$ of in-neighbors in $F$ is not empty. Pick $j$ such that $2^{j-1} \leq|X|<2^{j}$. Since $\left|Y_{j}\right| \leq 2^{j}$, by property $(*)$, family $\bar{S}_{j}$ contains a set $S_{j, i}$ that his $X$ and avoids $Y_{j}$. This $S_{j, i}$ will occur in one of the stages $s_{c}, s_{c}+1, \ldots, s_{c}+a_{j}$ -1 .

All in-neighbors of $v$ are either in $X$ or are inactive when stage $s_{c}$ starts. When we use $S_{j, i}$ for transmission, then

(i) Exactly one in-neighbor of $v$ in $X$ will transmit because $\left|S_{j, i} \cap X\right|=1$,

(ii) The nodes from $Y_{j}$ will not interfere because $S_{j, i} \cap Y_{j}$ $=\varnothing$, and

(iii) The nodes that were inactive at the beginning of stage $s_{c}$ and are not in $Y_{j}$ remain inactive when $S_{j, i}$ is issued, so they will not transmit.

Therefore, $v$ will receive the message when $S_{j, i}$ is issued (unless it has already received it earlier). Since $v$ was an arbitrary inactive out-neighbor of $F$, we conclude that all nodes in $F$ will become inner after $a_{g}$ stages.

Take $s_{c+1}=s_{c}+a_{g}$. In this case, $i_{c+1} \geq i_{c}+f_{c}$ and $f_{c} \geq 2^{g-1}$, so

$$
\begin{aligned}
& 2 i_{c+1}+f_{c+1} \geq 2\left(i_{c}+f_{c}\right)+0=2 i_{c}+f_{c}+f_{c} \\
& \geq \frac{s_{c}}{2 \alpha \log n}+2^{g-1} \geq \frac{s_{c+1}}{2 \alpha \log n} .
\end{aligned}
$$

We thus proved that (1) holds for $s_{c+1}$. Further, since $|F| \leq$ $k$, we have $a_{g}=\alpha 2^{g} \log n \leq 2 \alpha k \log n$, and, thus, in both subcases, we have $s_{c+1}-s_{c} \leq 2 \alpha k \log n$. Thus, the proof of the lemma is now complete.

\section{DISTRIBUTED COUPON COLLECTION}

In each phase of our algorithm, we will attempt to distribute each message $m_{v}$ to some number of nodes, by performing a sequence of limited broadcasts. We need to achieve two goals. To obtain a good running time, the number of limited broadcasts must be small. Further, each message $m_{v}$ should participate in at least one limited broadcast, that is, $m_{v}$ must be in at least one $M_{u}$, for some $u$ that initiates a limited broadcast.

To choose the nodes $v$ for which we initiate a limited broadcast, we use randomization. The principle behind the random process that we use is similar to that in the coupon collector's problem. There are two differences though. First, each coupon may have several copies. Second, since we do not have enough time to coordinate the choices, we cannot guarantee that exactly one node will initiate broadcasting.

We think of $V$ as a set of $n$ bins and $M$ as a set of $n$ coupons. Each coupon has at least $k$ copies, each copy belonging to a different bin. $M_{v}$ is the set of coupons in bin $v$. Consider the following process: At each step, we open bins at random, by choosing each bin, independently, with probability $1 / n$. If exactly one bin, say $v$, is opened, all coupons from $M_{v}$ are collected. If no bin is opened, or if two or more bins are opened, a failure occurs and no coupons are collected. How many steps do we need so that with high probability (a copy of) each coupon is collected?

The distributed coupon collection can be written in pseudocode as follows:

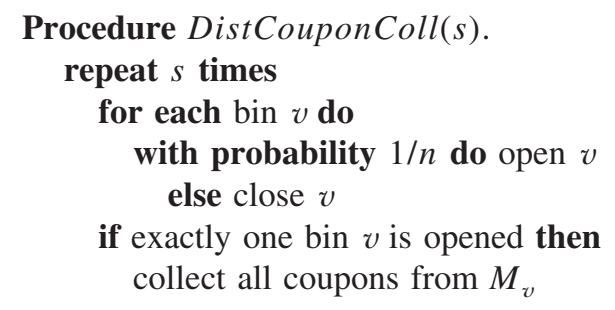

Lemma 2. Assume that we have $n$ bins and $n$ coupons and that each coupon has at least $k$ copies, each copy belonging to a different bin. Let $\delta$ be a given constant, $0<\delta<1$, and $s=(4 n / k) \ln (n / \delta)$. Then, after performing DistCoupon$\operatorname{Coll}(s)$, with probability at least $1-\delta$, all coupons will be collected.

Proof. The lemma is trivially true for $n=1$, so we can assume that $n \geq 2$. Let $\chi_{m, j}$ be the event that coupon $m$ is collected at a given step $j$. Then, $\operatorname{Pr}\left[\chi_{m, j}\right]$ is the probability that one bin containing $m$ is opened and all other bins are closed.

For all $m$ and $j$, we have

$$
\operatorname{Pr}\left[\chi_{m, j}\right] \geq \frac{k}{n}\left(1-\frac{1}{n}\right)^{n-1} \geq \frac{k}{n}\left(1-\frac{1}{n}\right)^{n} \geq \frac{k}{4 n},
$$

where the last inequality follows from the fact that the sequence $(1-1 / n)^{n}$ is monotonely increasing. The probability that some $m$ is not collected in $s$ steps is

$$
\operatorname{Pr}\left[\bigvee_{m \in M} \wedge_{j \leq s} \neg \chi_{m, j}\right] \leq n\left(1-\frac{k}{4 n}\right)^{s} \leq n e^{-s k / 4 n} \leq \delta,
$$

by the definition of $s$.

\section{THE GOSSIPING ALGORITHM}

We now present our Monte Carlo algorithm for gossiping. Each node $v$ performs its version of the algorithm: 


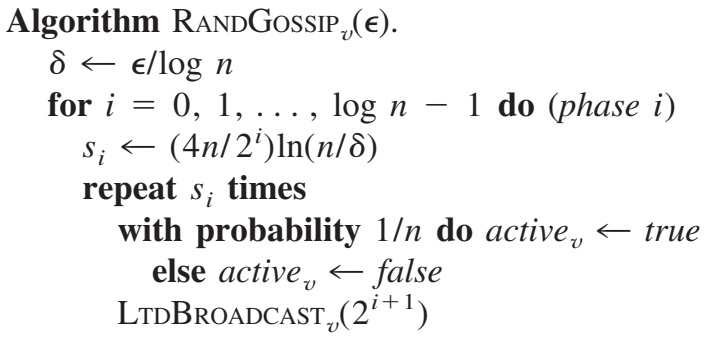

Theorem 1. Let $\epsilon, 0<\epsilon<1$, be a given constant. With probability at least $1-\epsilon$, Algorithm $\operatorname{RANDGossip}(\epsilon)$ completes gossiping in time $O\left(n \log ^{3} n \log (n / \epsilon)\right)$.

Proof. In phase $i$, the call to $\operatorname{LtDBROADCAST}_{y}\left(2^{i+1}\right)$ costs $O\left(2^{i} \log ^{2} n\right)$, so phase $i$ costs $O\left(s_{i} 2^{i} \log ^{2} n\right)=O(n$ $\left.\log ^{2} n \log (n / \delta)\right)$. Since we have $\log n$ phases and $\delta=\epsilon / \log$ $n$, this implies the bound on the running time of the algorithm.

Initially, when phase 0 starts, each $m_{u}$ is in one set $M_{v}$, namely, in $M_{u}$. The algorithm attempts to maintain the invariant that after phase $i$ each $m_{u}$ is in at least $2^{i+1}$ sets $M_{v}$. If this invariant is preserved at each phase, the gossiping will complete successfully, since after phase $\log n-1$, each $m_{u}$ will be in $n$ sets $M_{v}$. Thus, it is sufficient to prove that the probability that the invariant fails in some phase is at most $\epsilon$.

The process of distributing messages in a given phase $i$ is equivalent to the distributed coupon collection problem described in the previous section, where we view each node as a bin and active nodes correspond to open bins. Thus, by Lemma 2, the probability that the invariant fails in this phase, assuming that it has not failed in any previous phase, is at most $\delta$. So, overall, the probability of failure in some phase is at most $\log n \cdot \delta=\epsilon$.

To obtain a Las Vegas algorithm, run RANDGossip $(\epsilon)$ with $\epsilon=1 / n$. After the algorithm halts, each node that has not received $n$ different initial messages announces that a failure occurred. Since all these nodes send out the same message, this can be achieved with just one broadcast. If $B$ is the running time of the broadcasting algorithm, after $B$ steps, each node knows whether the gossiping was successful or not. If the gossiping failed, we run a naive, deterministic RoundRoBIN algorithm that consists of $n$ rounds, with each node transmitting once in each round. This will achieve gossiping in time $O\left(n^{2}\right)$. Overall, the expected running time will be $O\left((1-1 / n) n \log ^{4} n+(1 / n) n^{2}\right)$ $=O\left(n \log ^{4} n\right)$. Concluding, we get the following theorem:

Theorem 2. There exists a randomized Las Vegas algorithm for gossiping with expected running time $O\left(n \log ^{4} n\right)$.

\section{FINAL COMMENTS}

Several open problems remain: The only known lower bounds for gossiping are those for broadcasting [1, 5, 8], a seemingly easier problem. The gap between lower and upper bounds is particularly wide in the deterministic case: between $\Omega(n \log n)$ and $O\left(n^{3 / 2} \log ^{2} n\right)$. Closing or at least reducing this gap is an interesting open problem.

Our algorithm is probably not optimal. One possible research direction is to investigate whether one can improve the running time of our algorithm by using the randomized broadcasting algorithm from [3] to perform limited broadcast. It is not quite clear whether the algorithm from [3] can be modified to satisfy Lemma 1. Further, the analysis of this modified algorithm will probably be much more complicated.

Improving the bounds on gossiping or broadcasting may resolve the question whether gossiping is harder than is broadcasting in the radio network model (both in the randomized and the deterministic case). Our result implies that, at least in the randomized case, the difference is at most a polylogarithmic factor.

\section{Acknowledgments}

We would like to thank the anonymous referees for useful comments that helped us improve the presentation. This research was done while the first author was visiting the University of Liverpool.

Note added in proof. D. Kowalski and A. Pelc showed recently that the lower bound of $\Omega(n)$ in [13] mentioned in the introduction is not valid. See [D. Kowalski and A. Pelc, Deterministic broadcasting time in radio networks of unknown topology, in Proc., 43rd Annual IEEE Symposium on Foundations of Computer Science, 2002, pp. 63-72.]

\section{REFERENCES}

[1] N. Alon, A. Bar-Noy, N. Linial, and D. Peleg, A lower bound for radio broadcast, J Comput Syst Sci 43 (1991), 290-298.

[2] N. Alon, A. Bar-Noy, N. Linial, and D. Peleg, Single round simulation of radio networks, J Alg 13 (1992), 188-210.

[3] R. Bar-Yehuda, O. Goldreich, and A. Itai, On the time complexity of broadcast in radio networks: An exponential gap between determinism and randomization, J Comput Syst Sci 45 (1992), 104-126.

[4] R. Bar-Yehuda, A. Israeli, and A. Itai, Multiple communication in multi-hop radio networks, SIAM J Comput 22 (1993), 875-887.

[5] D. Bruschi and M. del Pinto, Lower bounds for the broadcast problem in mobile radio networks, Distrib Comput 10 (1997), 129-135.

[6] I. Chlamtac and S. Kutten, On broadcasting in radio networks-problem analysis and protocol design, IEEE Trans Commun 33 (1985), 1240-1246.

[7] B.S. Chlebus, L. Ga̧sieniec, A.M. Gibbons, A. Pelc, and W. Rytter, Deterministic broadcasting in unknown radio networks, Proc 11th Ann ACM-SIAM Symp on Discrete Algorithms, San Francisco, CA, 2000, pp. 861-870. 
[8] M. Chlebus, L. Gassieniec, A. Östlin, and J.M. Robson, Deterministic broadcasting in radio networks, Proc 27th Int Colloq on Automata, Languages and Programming, Lecture Notes in Computer Science, Vol. 1853, Springer, 2000, pp. 717-728.

[9] M. Christersson, L. Ga̧sieniec, and A. Lingas, Gossiping with bounded size messages in unknown ad hoc radio networks, manuscript, 2002.

[10] M. Chrobak, L. Gassieniec, and W. Rytter, Fast broadcasting and gossiping in radio networks, 41st Ann IEEE Conf on Foundations of Computer Science, FOCS'00, 2000, pp. $575-581$

[11] G. De Marco and A. Pelc, Faster broadcasting in unknown radio networks, published manuscript, 1999.

[12] K. Diks, E. Kranakis, D. Krizanc, and A. Pelc, The impact of knowledge on broadcasting time in radio networks, Proc 7th European Symp on Algorithms, 1999, pp. 41-52.

[13] A. Ephremides and B. Hajek, Information and communication networks: An unconsummated union, IEEE Trans Info Theory IT-44 (1998), 2416-2434.

[14] I. Gaber and Y. Mansour, Broadcast in radio networks, Proc 6th Ann ACM-SIAM Symp on Discrete Algorithms, 1995, pp. 577-585.

[15] R. Gallager, A perspective on multiaccess communications, IEEE Trans Info Theory IT-31 (1985), 124-142.

[16] S.T. Hedetniemi, S.T. Hedetniemi, and A.L. Liestman, A survey of gossiping and broadcasting in communication networks, Networks 18 (1988), 319-349.

[17] J. Komlos and A.G. Greenberg, An asymptotically non- adaptive algorithm for conflict resolution in multiple-access channels, IEEE Trans Info Theory IT-31 (1985), 302-306.

[18] E. Kranakis, D. Krizanc, and A. Pelc, Fault-tolerant broadcasting in radio networks, Proc 6th European Symp on Algorithms, Venice, Italy, 1998, Lecture Notes in Computer Science 1461, Springer, pp. 283-294.

[19] E. Kushilevitz and Y. Mansour, An $\Omega(D \lg (N / D))$ lower bound for broadcast in radio networks, SIAM J Comput 27 (1998), 702-712.

[20] E. Kushilevitz and Y. Mansour, Computation in noisy radio networks, Proc 9th Ann ACM-SIAM Symp on Discrete Algorithms, 1998, pp. 236-243.

[21] J.L. Massey and P. Mathys, The collision channel without feedback, IEEE Trans Info Theory IT-31 (1985), 192-204.

[22] R. Motwani and P. Raghavan, Randomized Algorithms, Cambridge University Press, Cambridge, 1995.

[23] K. Pahlavan and A. Levesque, Wireless Information Networks, Wiley-Interscience, New York, 1995.

[24] D. Peleg, Deterministic radio broadcast with no topological knowledge, manuscript, 2000.

[25] K. Ravishankar and S. Singh, Asymptotically optimal gossiping in radio networks, Discr Appl Math 61 (1995), 6182.

[26] K. Ravishankar and S. Singh, Gossiping on a ring with radios, Parallel Process Lett 6 (1996), 115-126.

[27] A. Sen and M.L. Huson, A new model for scheduling packet radio networks, Proc 15th Ann Joint Conf of the IEEE Computer and Communication Societies, 1996, pp. 11161124. 